\title{
Informationswissenschaft
}

Jan Brademann*

\section{Für eine informationswissenschaftliche Aufwertung von Rezensionen}

DOI 10.1515/iwp-2017-0001

Zusammenfassung: Der Autor plädiert dafür, wissenschaftliche Rezensionen stärker als Gegenstand informationswissenschaftlicher Forschung und Praxis in den Blick zu nehmen. Verabschiedet man sich von der Vorstellung, dass dokumentarische Objektivität durch die Minimierung der Subjektivität des Abstracters zu erreichen sei, und begreift man (intellektuelles) Abstracting als produktiven Prozess, der wesentlich von den sozialen Regeln der scientific community bestimmt ist, so lässt sich erkennen, dass das Interesse an wissenschaftlichen Rezensionen als spezifischer Textsorte Teil eines gruppenspezifischen Informationsverhaltens ist.

Deskriptoren: Referat, Referieren, Informationsanalyse, Dokumentation, Kritik, Informationsverhalten

\section{Reassessment of scientific reviews by the information science}

\begin{abstract}
The author recommends scientific reviews to be treated as an integral part of information science and practice. It suggests moving away from the current idea that documental objectivity can be gained by reducing the abstractor's subjectivity. Instead, we ought to understand abstracting as a productive process, determined by the rules of the scientific community. Seen in this light, scientific reviews may attract a new interest as a part of a specific form of information behaviour.
\end{abstract}

Descriptors: Abstract, Abstracting, Information analysis, Documentation, Criticism, Information behaviour

\section{Pour une revalorisation des comptes rendus scientifiques au niveau des sciences de l'information}

Résumé: L'auteur recommande d'utiliser plus souvent des comptes rendus scientifiques comme partie intégrante de la recherche et la pratique en sciences de l'information. Si

*Kontaktperson: Dr. Jan Brademann, Evangelische Landeskirche Anhalts, Landeskirchliches Archiv, Friedrichstraße 22/24, 06844 Dessau-Roßlau, E-Mail: jan.brademann@kircheanhalt.de on se détache de l'idée que l'objectivité documentaire peut être atteinte en minimisant la subjectivité de la personne qui fait le compte rendu, et si on prend l'analyse (intellectuelle) comme un processus productif, qui est en grande partie déterminée par les règles sociales de la communauté scientifique, alors on peut voir que l'intérêt pour les comptes rendus scientifiques en tant que type spécifique de texte fait partie d'un comportement de l'information spécifique à un groupe.

Descripteurs: Présentation, Référencement, Analyse de l'information, Documentation, Critique, Comportement en matière d'information

„Rezensionen“ sind ein komplexes Phänomen der Wissens- und Informationsgesellschaft. Unter dem Terminus kann ganz Unterschiedliches verstanden werden: Als Kundenrezensionen sind zumeist kurze, kaum formalisierte Meinungsäußerungen einzelner User im Internet $\mathrm{zu}$ Produkten oder Dienstleistungen in Textform zu verstehen. ${ }^{1}$ Ihnen gegenüber stehen wissenschaftliche Rezensionen, die, in wissenschaftlichen Zeitschriften oder auf Internetseiten wissenschaftlicher Einrichtungen veröffentlicht, Ergebnisse der Forschung zum Gegenstand haben. Aus sprachwissenschaftlicher Sicht sind sie als eine Fachtextsorte zu definieren: Sie sind „öffentliche, monologische Texte“, Texte, ,in denen ein wissenschaftlich relevanter Rezensionsgegenstand beschrieben und bewertet wird“. ${ }^{2}$ Hinzufügen lässt sich eine große Gruppe von Rezensionen in unterschiedlichsten Medien, die einer breiten Leserschaft Informationen über wissenschaftliche, nichtoder populärwissenschaftliche Literatur bieten. Zusammen bilden sie die meistverbreitete Form der „Literaturkritik".3

Dem Ursprungswortstamm - lat. recensio $=$ Musterung, bzw. recensere = prüfen, in Augenschein nehmen folgend, ist Rezensionen gemein, dass sie auf ein Objekt

1 Kastner/Mandl/Straß 2013, S. 386.

2 Ripfel 1998, S. 491.

3 Albrecht 2001, S. 53. 
referenzieren. Diese Funktion macht sie für die Informationswissenschaft, und hier insbesondere die Wissensrepräsentation, interessant. Ganz unterschiedlich ausgeprägt ist freilich die Art der Referenz: Während Kundenrezensionen typischerweise auf eine subjektive Bewertung beschränkt sind - weswegen sie für Verfahren des OpinionMining in den Blick geraten,$-{ }^{4}$ sind wissenschaftliche und populärwissenschaftliche Rezensionen als Mischform aus einer „objektiven“ Beschreibung des Objekts, seiner „subjektiven“ Bewertung und weiterer (Meta-)Informationen einzuschätzen, wobei diese Elemente unterschiedlich gewichtet sind. ${ }^{5}$

Hier liegt der Ansatzpunkt für die folgende Argumentation. Wissenschaftliche Rezensionen sind „Sekundärtextsorten“, „wissensreproduzierende Fachtextsorten“: Sie informieren über einen Primärtext, also potenziell dokumentarische Bezugseinheiten (DBE), bewegen sich aber in einem Grenzbereich zwischen Referat und Aussagen zusätzlichen Informationsgehalts. Was bedeutet diese Zwischenstellung für die Informationswissenschaft? Inwiefern sollte sie Rezensionen als Dokumentationseinheiten (DE) verstehen und analysieren? Die folgenden Überlegungen können nur einige Denkanstöße zu diesem Thema geben, indem sie Methoden anderer Disziplinen zu rezipieren und deren spezifische Informationsinteressen heuristisch zu berücksichtigen suchen. Ich gehe dabei in drei Schritten vor: Zunächst versuche ich, die Forschungssituation zu klären (1.). Anschließend widme ich mich dem Kriterium der Objektivität (2.), das wesentlich dafür verantwortlich ist, dass Rezensionen bislang kein größeres Interesse der Informationswissenschaft beanspruchen. Da dies vor allem deswegen problematisch ist, weil Objektivität nur als intersubjektiv stabilisierte Subjektivität realisiert werden kann, versuche ich danach, Rezensionen als eine fachspezifische Textsorte zu modellieren (3.), deren Erforschung Teil eines größeren Forschungsdesigns sein könnte, das sich fachspezifischem information behaviour widmet - nicht zuletzt als Grundlage für ebenso fundierte wie gezielte Dokumentationsarbeit.

4 Kastner/Mandl/Straß 2013.

5 Klauser 1992, S. 115-120. Vor allem die Germanistik hat sich den Funktionen und der Makrostruktur von Rezensionen gewidmet; dabei herrscht jedoch Uneinigkeit über die Gewichtung dieser Funktionen und damit den Hauptzweck von Rezensionen; vgl. Dzika 2007, S. 88.

\section{Ein weißer Fleck der Informationswissenschaft?}

Schaut man in informationswissenschaftliche Hand- und Lehrbücher, so entsteht der Eindruck, dass die Rezension ihren Ort innerhalb dieser Wissenschaft noch sucht. Während sie in vielen Veröffentlichungen schlichtweg nicht vorkommt, ${ }^{6}$ wird sie etwa von Wilhelm Gaus im Zusammenhang mit den Techniken und Methoden des Erschließens erörtert ${ }^{7}$ und von Wolfgang Stock in dem Kapitel zu „Kurzreferate[n] als Methode der Informationsverdichtung “ am Rand erwähnt. ${ }^{8}$

Die Deutungen beider Autoren gehen jedoch in ganz unterschiedliche Richtungen: Gaus stuft die Rezension als ein Abstract, genauer gesagt „informatives Referat“, ein, das um ,eine persönliche (subjektive) Stellungnahme oder Beurteilung erweitert" sei. ${ }^{9}$ Von Stock hingegen werden Rezensionen als Texte klassifiziert, die sich von Referaten grundlegend unterscheiden: Bereits das ,bewertende Abstract“ ist aus seiner Sicht als „Grenzform eines Abstracts zum Einsatz in der Wissensrepräsentation“ zu charakterisieren. Es verstoße nämlich „gegen die Vorschrift der Objektivität“, die unter anderem die DIN-Norm 1426 an Abstracts stellt. Daher sei das bewertende Abstract weniger mit dem echten Abstract als mit Literaturberichten und Rezensionen verwandt, denen keine Funktion zur Wissensrepräsentation zukomme. ${ }^{10}$ Auch Rainer Kuhlen sieht in seinem Handbuchartikel zum Referieren in Anlehnung an DIN 1426 Auszug, Zusammenfassung, Annotation und Kurzreferat als für die Dokumentation „einschlägige“ Formen von Abstracts an und blendet Rezensionen und Sammelrezensionen sowie den Literaturbericht aus, obschon auch diese in jener DIN genannt sind. ${ }^{11}$

Inwiefern hinter diesen Positionen wissenschaftsgeschichtliche Veränderungen stehen - ein Autor wie Gaus, Jahrgang 1939, gehört als Psychologe und späterer Professor für medizinische Statistik, Dokumentation und Informatik einer anderen Generation als Stock und Kuhlen an -, muss an dieser Stelle dahingestellt bleiben. Eine mögliche Erklärung dieser unterschiedlichen Einstufungen der Textsorte Rezension liegt in unterschiedlichen Fragerichtungen der Wissenschaftler: Gaus argumentiert vom Erschließen durch eine Fachbibliothek als einer Lite-

\footnotetext{
6 Vgl. etwa Kuhlen 2013; Sparck-Jones/Willett 1997.

7 Gaus 2003.

8 Stock/Stock 2008, S. 390.

9 Gaus 2003, S. 52.

10 Stock/Stock 2008, S. 390.

11 Kuhlen 2004, S. 193.
} 
raturdokumentationsstelle her. Er hat die Belange der Fachinformation im Visier, die aus seiner Sicht im Übrigen auch den Dokumentar dazu zwingen, nicht nur die strukturale (klassifikatorische), sondern auch die inhaltliche Dimension des Fachgebiets ,seiner‘ Dokumente „recht gut [zu] beherrschen". ${ }^{12}$

Könnte nicht vielleicht, so ist an dieser Stelle zu fragen, aus einer so hergeleiteten Notwendigkeit zur fachspezifischen Urteilsfähigkeit des Dokumentars eine größere Aufgeschlossenheit gegenüber der Textsorte Rezension fließen? Grundlegend anders scheint dies jedenfalls dort zu sein, wo es in erster Linie um die überall und gegenüber jedermann zu gewährleistende Verfügbarkeit elektronisch gespeicherten Wissens geht. Dieser Interessensschwerpunkt entspricht in der Tat einer jüngeren Phase der Informationswissenschaft. Unter dem ingenieurswissenschaftlichen Primat einer systemorientiert-technischen Sicht auf Information müssen die Dokumentare Spezialisten der Informationsarbeit, aber keine Fachleute mehr sein..$^{13}$ Auch Wolfgang Stocks Interesse am (echten) Abstract rührt von der Repräsentation, Organisation und Ordnung von Wissen innerhalb eines digitalen Systems: DBE sollen durch Surrogate präzise, objektiv und eindeutig „abgebildet“ sein, um so „optimal gesucht und gefunden [zu] werden“. ${ }^{14}$ Das Retrieval wird primär durch Metadaten, sekundär durch Begriffe und erst in dritter Hinsicht durch Aussagen beeinflusst.

Der gewichtigste Unterschied zwischen solchen Abstracts und wissenschaftlichen Rezensionen dürfte in der Textproduktion $\mathrm{zu}$ suchen sein: Während erstere (wenn auch nicht ausschließlich) als Produkte der Tätigkeit von Dokumentaren einzuschätzen sind, ist die Entstehung von wissenschaftlichen Rezensionen innerhalb derjenigen Wissenschaft, deren Produkt die Referenzeinheit ist, selbst $\mathrm{zu}$ verorten, wenngleich die Intentionen zum Schreiben ökonomischer oder politischer Art sein können. ${ }^{15}$ Gleich-

12 Gaus 2003, S. 48. Dass Gaus in der Tat jener Typus des Dokumentars vorschwebt, der sich seit der Mitte des 20. Jahrhunderts durchsetzte, kann hier nur angedeutet werden: Nachdem das traditionelle Bibliothekswesen von fachfremdem (zumeist geisteswissenschaftlich sozialisiertem) Personal dominiert worden war, konstituierte sich Dokumentation als Arbeitsfeld seit den 1920er Jahren gerade auch in dem Anspruch, dokumentarische Arbeit durch fachwissenschaftlich geschultes Personal zu bieten - und so den Nutzer nicht bloß auf Dokumente zu verweisen, sondern diese selbst $\mathrm{zu}$ erschließen, kritisch zu werten und für den Nutzer zusammenzustellen; vgl. Seeger 2013, S. 27-29.

13 Ebd., S. 32-34.

14 Stock/Stock 2008, S. XI. Stock und Stock vertreten einen engeren Begriff der Wissensrepräsentation, indem sie sie per definitionem an „digitale Datenbanken“ binden. wohl bleibt das Desinteresse der Informationswissenschaft erklärungsbedürftig, weil sie in ihrem Erklärungsanspruch nicht auf die Arbeit von Dokumentaren beschränkt ist, sondern viel weiter reicht, indem sie Wissen in Kontext und Aktion, seine Speicherung, Beschreibung, Zugänglichmachung und Kommunikation erklären und optimieren will.

\section{Automatismen und die Fiktion der Objektivität}

Schauen wir noch einmal auf die obigen Argumentationen, so schälen sich die ,objektiven' Gehalte der Texte als das entscheidende Kriterium heraus, mittels dessen diese beurteilt werden. Dass sich dies für die Germanistik umgekehrt darstellen kann, kann ich hier nur andeuten. ${ }^{16}$ Mit zwei Mitteln wird nun postuliert, die „Aboutness“ des Dokuments, „das, worüber es geht“, eindeutig fixieren zu können: erstens durch die Überzeugung, zu einer objektiven Repräsentation des expliziten Wissens der DBE gelangen, zweitens durch den Anspruch, das Abstracting durch Regelgeleitetheit institutionalisieren zu können. So schreibt die DIN 1426 Vollständigkeit (der zentralen inhaltlichen Aspekte der DBE), Genauigkeit (präzise Wiedergabe der Terminologie und Thesen der DBE), Objektivität (keine expliziten Wertungen des Abstracters) sowie Kürze und Verständlichkeit des Abstracts vor. ${ }^{17}$ Darüber hinaus hat sich die Würdigung der zentralen strukturellen Bestandteile des Textes - Hypothese, Zielsetzung, Gegenstand, Verfahren und Methode, Ergebnis, Schlussfolgerung, Anwendung, Zeitraum und geographischer Raum - als Konsens etabliert. ${ }^{18}$

Bereits diese Ordnungsansprüche sind freilich unscharf - wie bemisst sich Vollständigkeit von Repräsentation? -, ja in sich widersprüchlich: Widerstreiten nicht die Prinzipien Vollständigkeit und Kürze? Die Frage nach der Aboutness wird unten zu diskutieren sein; an dieser Stelle sei verwiesen auf William John Hutchins, einem Vertreter der textlinguistisch basierten Theorie des Abstractings, auf den sich auch Wolfgang Stock bezieht. Hut-

15 Liang 1999, S. 292, führt sieben Gründe für die Entstehung von Rezensionen an.

16 Fokussiert sie auf die eigenständige Kritik des Rezensenten als eigentlich wichtigem Merkmal dieser Textsorte, so dreht sich das Verhältnis von Abstract und Rezension nachgerade um. Daher geraten die Anzeige (Kurzrezension) und das Referat zu Unterformen der Rezension; Klauser 1992, S. 51.

17 Kuhlen 2004, S. 196 f.

18 Ebd., 197. 
chins betont, dass die Aboutness, die durch Abstracting dargestellt werden soll, diejenige des Textes per se sein und gegenüber Nutzern jedweden Profils dargestellt werden solle. Sie könne nur identifiziert werden, wenn „der Indexer nicht sein eigenes Bild vom Text an irgendwelche Nutzer heran[trägt], sondern jede Beeinträchtigung zwischen Dokument und Leser minimier[t] “. ${ }^{19}$ Um zu Objektivität zu gelangen, müsse also der Abstracter bemüht sein, seine Subjektivität zu minimieren. Aus einer solchen Sicht folgt konsequenterweise das Credo, dass Indexing und Abstracting von Maschinen ebenso wie von Menschen übernommen werden können. ${ }^{20}$

In der Tat scheint sich die Informationswissenschaft vor allem in die Richtung einer vom Subjekt des Dokumentars abstrahierenden, automatisierten Informationsaufbereitung zu bewegen. Informationsorientiertes Schreiben wird als regelgesteuerte Tätigkeit von reinen Informationsspezialisten im Schnittfeld von sprachlicher Kompetenz und technischer Unterstützung konzipiert. ${ }^{21}$ Die Erforschung der Potenziale automatischer Verfahren des Abstractings boomt. Dies ist angesichts der Kenntnis der Grenzen dieser Verfahren besonders interessant: Diese liegen gerade dort, wo das Abstracting über das Isolieren und Selektieren von relevanten Einzelinformationen hinausgeht. Automatisches Abstracting wird dadurch als eigene Form des Referierens modelliert, die andere Vorzüge als intellektuelles Abstracting besitzt, indem es etwa MehrDokumenten-Zusammenfassungen im Kontext fluider Publikationsformen ermöglicht. ${ }^{22}$ Zugleich gerät aber das intellektuelle Abstracting mit seinen Vorzügen ein stückweit aus dem Blick.

Diese Forschungstendenz erklärt sich gewiss aus der Dominanz elektronischer und digitaler Daten- und Ordnungssysteme für die dokumentarische Arbeit, die darauf abzielt, angesichts steigender Datenmengen eine allgemeine Kompetenz des information retrieval zu gewährleisten. ${ }^{23}$ Als maßgeblich hinzu kommt aber auch das oben angesprochene Objektivitätsideal. Dieses ist aber als

19 Stock/Stock 2008, S. 35.

20 Ebd., S. 33.

21 Zahlreiche Bemühungen um eine Optimierung informationsorientierten Schreibens haben ihre Impulse aus den veränderten Rahmenbedingungen elektronischen Publizierens bekommen und suchen in technischen, soziologischen und linguistischen - und gerade nicht literarischen oder geisteswissenschaftlichen (hermeneutischen) -Fächern nach Anregungen; vgl. Endres-Niggemeyer 1998; Endres-Niggemeyer 1988.

22 Hahn 2013, S. 286-301.

23 Die Impulse der Computerlinguistik, deren Methoden sich angesichts steigender Datenmengen „vom Sprachverstehen zur Sprachtechnologie“ verschieben, führt an: Hahn 2004.
Objektivitätsfiktion zu kennzeichnen, weil es letztlich keine symbolische Repräsentation und Kommunikation von Sachverhalten unabhängig von Bedeutungszuweisung und damit immer auch Bewertung - geben kann. Dieses empiristisch-kulturalistische Credo ist keineswegs als Totschlagsargument $\mathrm{zu}$ verstehen, sondern soll neue Perspektiven eröffnen: Objektivität ist als Regulativ der Forschung unverzichtbar, denn sie definiert, was als Wahrheit anerkannt werden darf und was nicht. Sie sollte aber nicht als Merkmal eines Seienden oder „Dinges an sich“ beschrieben werden, das außerhalb menschlicher Wahrnehmung existieren würde. Der Versuch des Forschers oder eben auch des Dokumentars, bei der Interpretation von Objekten - hier Forschungsergebnissen und -material bzw. Wissen von DBE - die eigene Subjektivität zu minimieren, muss immer unvollständig bleiben. Daher kann Objektivität als wissenschaftliche Allgemeingültigkeit nur dadurch erreicht werden, dass man reflektiert und nachvollziehbar mit Subjektivität umgeht. Sie kann nur als eine intersubjektiv stabilisierte Subjektivität vorgestellt werden. Ihre Kriterien sind nicht transzendental, sondern sozial bestimmt: Die scientific community legt fest, woran sich „Wahrheit“ messen lassen muss. ${ }^{24}$

Und so dürften auch die Kriterien, mit denen die Informationswissenschaft Abstracts kennzeichnet, als analytische Instrumente für die Makrostruktur von Texten und als Richtschnur für die Dokumentationsarbeit zu verstehen sein. Als Argumente, Rezensionen als DE auszuschließen, taugen sie hingegen weniger: Alle Prozesse der Informationsverdichtung sind kognitive Vorgänge, die von der Intention, der sprachlichen und fachlichen Fähigkeit sowie der sozialen Prägung des Dokumentars bzw. der Dokumentarin determiniert werden. Bewertung findet nicht nur dort statt, wo sie sprachlich hervorgehoben wird. Auch vermeintlich objektive Paraphrasen entpuppen sich als Wertungen: Die Evaluativität von Propositionen erschließt sich oft erst aus dem sozialen Kontext, aus dem Zeichenvorrat und semantischen Codes der Gruppe. ${ }^{25}$ Bereits die Feststellung der Referierwürdigkeit einer DBE ist vom Standort des Dokumentars abhängig. Mit der „Abstraktion" (lat. abstractio = Herausnahme) bestimmter als relevant eingeschätzter, zuungunsten anderer, unwesentlicher Vorstellungs- und Begriffsinhalte wird immer auch Wissen ausgeschieden. ${ }^{26}$ Die im Prozess der kognitiven

24 Die Literatur zu diesem erkenntnistheoretischen Komplex ist Legion; hier muss es genügen, auf das Kapitel „objektiv/subjektiv“ zu verweisen in dem bewährten Buch von Daniel 2001, S. 368-397.

25 Dzika 2007, im Hinblick auf Rezensionen.

26 Mit der Möglichkeit, die Aboutness im Hinblick auf das Abstract einzuschränken, der „paramorphen Informationsverdichtung“, die 
Informationsverdichtung identifizierte Aboutness ist folglich stets ein Produkt von Vorgängen der Interpretation und Diskriminierung durch ein sozial geprägtes Subjekt.

Doch um das Abstracting zu vervollständigen, ist ein Prozess nötig, der die selektierten Wissensbestände zu einem kohärenten und kohäsiven Ganzen zusammensetzt. Würde sich die DE auf Extraktion beschränken, wäre sie nicht Abstract (man hat freilich den Begriff des extraktiven Abstracts eingeführt), sondern ein Extract. Spätestens hier lauern die Tücken - für die Verfechter des automatisierten Abstracting wie für die Argumentation der Subjektivitätsminimierung: $\mathrm{Zu}$ abstrahieren heißt nämlich nicht nur, Sachverhalte zu isolieren, sondern auch zu generalisieren, also das einer Menge von Dingen und Vorstellungsinhalten Gemeinsame zu beschreiben. ${ }^{27}$ Und selbst wenn der Abstracter der Maßgabe von Hutchins folgt, nur solche Begriffe zu verwenden, die in der DBE zu finden sind, so gilt doch: Die Herstellung der syntaktischen und intersentenziellen Zusammenhänge zwischen den selektierten Bedeutungsgehalten schafft neue Bedeutungen - und führt letztlich immer zu einem neuen Text. Spätestens hier dürfte man der Grenzen des Abstractings als eines mechanischen Prozesses gewahr werden; spätestens hier greifen die Argumente derjenigen, für die Abstracting eine Kunst ist. $^{28}$

\section{Rezensionen als gruppenspezifische Textsorte}

Auch Abstracts, die mehr sind als maschinelle Extracts, sind also auf der einen Seite als subjektiv zu kennzeichnen. Nun ist aber zu fragen, ob Rezensionen auf der anderen Seite wirklich so subjektiv - und das meint: individuell - sind, dass sie dem Objektivitätsideal mehr widersprechen als übrige Formen von Abstracts. Gewiss, explizite Normierungen fehlen. Dennoch lassen sich fach-

zu einem „perspektivische[n] Kurzreferat“ führt, indem Teile der Aboutness weggelassen werden, ist diese Diskriminierung frappierend. Vgl. Stock/Stock, 2008, S. 383-385.

27 Acham 1971. In der Informationswissenschaft hat sich freilich die Differenzierung zwischen extraktivem (Abstracting als Extracting) und derivativem Abstracting durchgesetzt.

28 Kuhlen 2004, S. 191 f. Intersentenzielle Verknüpfung und inhaltsbezogenes Generalisieren bilden für die Bemühungen um eine Optimierung automatischer Verfahren wahrscheinlich das größte Hindernis; ebd., S. 199-201. Nach Hahn 2013, S. 298, lautet die entscheidende Forschungsfrage, inwieweit „sich das automatische Abstracting vom sprachbehafteten extraktiven zum inhaltsbezogenen derivativen Abstracting weiter entwickeln kann“. spezifische Regelmäßigkeiten feststellen, die für das Verfassen und die Akzeptanz von Rezensionen maßgeblich sind und die sich in der und durch die Praxis fortschreiben.

Der Soziologe Frank Bardelle spricht, in Adaption von Bourdieus Habitus-Theorie und aufbauend auf der empirischen Analyse von soziologischen Rezensionen, von der Ausprägung eines „rezensiven Habitus““. ${ }^{29}$ Auch die Germanistik hat soziokulturelle und fachliche Faktoren für die Konstituierung von gemeinsamen Grundmustern von Vertextungsstrategien bei Rezensionen herausgearbeitet. ${ }^{30}$ In einem durch Wissenschaftsinstitutionen und -akteure regulierten „rezensiven Feld“ werden die Ordnungsschemata der Wahrnehmung und Gestaltung wissenschaftlicher Produkte in der rezensiven Praxis immer wieder neu konstituiert, und auch der „rezensive Habitus“ strukturiert die Nutzung und Neuproduktion von Rezensionen und ihre Akzeptanz. ${ }^{31}$ Gleichzeitig ist die rezensive Praxis wissenschaftssoziologisch ein Teil des Austauschs von kulturellem Kapital, durch den der soziale Raum der Wissenschaft und insbesondere seine Hierarchien strukturiert werden.

Daraus folgt zweierlei: Rezensionen sind erstens viel weniger subjektiv als angenommen. Vielmehr sind sie als Teil desjenigen Prozesses zu charakterisieren, dem sie dienen: der intersubjektiven Konstitution wissenschaftlicher Objektivität. Zweitens sind nicht nur Rezensent und Rezensierter, Fachkollegenschaft, Forschungseinrichtungen, Herausgeber, Redakteure und Verlage als Akteure im „rezensiven Feld“ zu verstehen, sondern auch Dokumentations- und Fachinformationsstellen. Sie tragen eine Mitverantwortung an der Bereitstellung rezensiver Information wie an der Konstituierung einer auf der Basis breitestmöglicher intersubjektiver Kommunikation stabilisierten institutionellen Selbstkontrolle der Wissenschaft.

Rainer Kuhlen gibt in seinem Handbuchartikel zum Referieren das in dieser Hinsicht entscheidende Stichwort: Es sei nämlich aus seiner Sicht die pragmatische Eigenschaft von Abstracts, die ihre besondere „Beliebtheit“ ausmache. Die in ihrer gegenüber Klassifikationen und Thesaurus größeren Ausdrucksstärke begründete Referenzund Indikationsleistung von Rezensionen geht, folgt man Kuhlens Deutung, über das bloße information retrieval hinaus. Rezensionen bieten nämlich dem potenziellen Leser nicht nur eine Zeitersparnis, senken Sprachbarrieren ab und helfen dabei - und dies dürften eben für die Wis-

29 Bardelle 1989.

30 Sie lassen sich primär an der hierarchischen und sequentiellen Gestaltung der illokutiven und propositionalen Textstruktur festmachen; vgl. z. B. Liang 2001, S. 289-311.

31 Klingenböck 2013, S. 105-107. 
senschaft die entscheidenden Kriterien sein -, Wissen zu kumulieren und $\mathrm{zu}$ integrieren, sondern sie fordern „zum Handeln auf, entweder die Originaltexte $\mathrm{zu}$ ignorieren oder sie genauer zur Kenntnis zu nehmen“. ${ }^{32}$

Rezensionen dienen unterschiedlichen Zwecken. Neben einer erörternden und wertenden, die sie von jenen deutlich absetzen, erfüllen Rezensionen auch eine informierende und eine aktivierende Funktion, die sie mit Kurzreferaten bzw. Abstracts im engeren Sinne teilen. ${ }^{33}$ Blickt man auf die Geschichtswissenschaft als akademische Disziplin oder Fachgruppe, so wird deutlich, dass sich ihr Profil und ihre Wahrnehmung in der Vergangenheit immer wieder wandelten, ${ }^{34}$ dass heute aber gerade diese Referenzleistung $\mathrm{zu}$ dominieren scheint. Obschon die Akzeptanz digitalisierter Fachkommunikation in dieser klassischen Buchwissenschaft im Vergleich zu den datenintensiveren, naturwissenschaftlichen Disziplinen noch immer geringer ausgeprägt ist, hat doch aufgrund des Internets auch im Umgang mit dieser Textsorte ein tiefgreifender Wandel eingesetzt. ${ }^{35}$

Seine Ausmaße sind kaum abzusehen. Erkennbar aber ist, dass die Rezension als wissenschaftlicher Informationsträger keinesfalls an Bedeutung verliert, eher im Gegenteil. Digital publizierte Internetrezensionen sind für Historiker zu sehr schnell und bequem abrufbaren Informationsquellen über neue Produkte der Forschung geworden. Ihre Nutzung über Datenbanken wie clio-online, online-Zeitschriften wie sehepunkte, vor allem auch Mailinglisten wie H-Soz-Kult ist mittlerweile ein fester Bestandteil der Routinen weiter Teile der community geworden. Mit diesen Veränderungen steigen die Ansprüche an die Geschwindigkeit der Verfügbarkeit von Rezensionen und damit an den Neuigkeitswert ihrer Informationen. Das hat noch kaum absehbare Folgen für die Produktion, Redaktion und höchstwahrscheinlich auch die Makrostruk-

32 Kuhlen 2004, S. 190.

33 Dzika 2007, S. 88, mit weiterführender Literatur.

34 Entstanden am Beginn moderner Wissenschaftlichkeit in der Aufklärungszeit, veränderte sich das Profil von Rezensionen als Teil von Wissenschaftskulturen und Gesellschaften, aber auch das Verhalten der Nutzer von Rezensionen wandelte sich. Während beispielsweise im 18. Jahrhundert Rezensionen den Charakter von Referaten trugen, wurden Rezensionen im frühen 20. Jahrhundert streckenweise durch zum Teil polemische Werturteile - zu Medien der Ehrzuweisung innerhalb der scientific community beispielsweise der Historiker; vgl. Scheutz 2013, S. 63-86.

35 Die Geschichtswissenschaft hat sicherlich deswegen Rezensionen erst jüngst als Forschungsgegenstand erkannt. Vgl. die Mitteilungen des Instituts für Österreichische Geschichtsforschung 121 (2013) (Themenheft Rezensionswesen), insb. Scheutz/Sommerlechner 2013. tur dieser Texte - eine mögliche Forschungsperspektive, die an dieser Stelle nicht verfolgt werden kann. ${ }^{36}$

Schließlich sind Online-Rezensionen, zunehmend auch als ex-post (im PDF-Format) online gestellte Rezensionen, integraler Teil eines Prozesses der Vervielfältigung, Zerstreuung und Hybridisierung von Publikationsformen im Internet, indem Rezensionen in Plattformumgebungen zusammengeführt, für thematisches Browsing aufbereitet und mit Bibliothekskatalogen verknüpft werden. ${ }^{37}$ Durch Foren und Kommentarfunktionen entstehen ,kollaborative Formen zur Bewertung wissenschaftlicher Literatur“, ja letztlich „lebendige Rezensionen“. ${ }^{38}$

\section{Fachspezifisches Review behaviour?}

In der Informationswissenschaft gibt es Ansätze, die sich mit diesen Beobachtungen zusammenbringen lassen: Die Informationshermeneutik versteht - vielleicht macht sie mit dem Credo, Information sei Wissen in Aktion und Kontext, am konsequentesten Ernst - Informationen nicht als isolierte, sondern in der soziokulturellen Praxis konstituierte Wissensbestände. Die Hermeneutik als die Lehre des Auslegens von Texten vor dem Hintergrund des Sinnzusammenhangs ihrer Autoren (und/oder Lesers) nähert sich dem Sinn von Texten vermittels der Operation eines sich in den Autor (und/oder Leser) hineinfühlenden Verstehens. Hiervon ausgehend, zielt die Informationshermeneutik darauf ab, die soziokulturelle Bedingtheit der Auslegung von Wissen auf eine paradigmatische Weise zu berücksichtigen. Information existiert aus der Sicht etwa von Rafael Capurro nur im sozialen Kontext: ${ }^{39}$ Eine bestimmte Gruppe von Menschen teilt indem und weil sie kommuniziert (und dabei u.a. Informationen austauscht) - einen Zeichenvorrat und ein damit verbundenes Vorverständnis der Welt. Aus dem Miteinandersein von Fachleuten im Besonderen entstehen folglich ein fachlicher Zeichen- und Bedeutungsvorrat sowie ein fachlicher Fragehorizont. Erst vor deren Hinter-

36 Es könnte gefragt werden, inwieweit nicht die zunehmende Verfügbarkeit von Abstracts (Extracts) und Volltexten gerade das bewertende Element in den Rezensionen stärker ausprägt, oder ob, angesichts des zunehmenden Zeitdrucks auf Onlinepublikationen, der Referatscharakter von Rezensionen (wieder) zunimmt, und wieweit sich Online- und Papierrezensionen hieran ausdifferenzieren.

37 Kaden 2013, S. 509-519.

38 Vgl. Landes 2011, S. 669-671.

39 Maßgeblich Capurro 1986; die Forschungsentwicklung seither überblickt Capurro 2006, S. 527-533. 
grund kann daher ein bestimmtes Wissen als Information, als Fachinformation, gelten. Hier kommt die oben vertretene konstruktivistische Vorstellung wieder ins Spiel: Denn auch die Kriterien dafür, inwiefern die Antwort auf eine wissenschaftliche Frage nicht nur als objektiv und wahr, sondern auch noch als neu einzuschätzen ist, werden durch die Fachgemeinschaft in kommunikativen Prozessen immer wieder neu konstituiert.

Heuristisch folgt daraus, Wissensrepräsentation, -ordnung ${ }^{40}$ und -organisation fachspezifisch zu analysieren und die Arbeit der Dokumentare und Informationsarbeiter fachspezifisch auszurichten: Terminologien und Klassifikationen, aber auch Referate folgen nicht der Fiktion eines isoliert-objektiven Wissens in der DBE, das für anonyme, primär durch kognitive Strukturen geprägte Nutzer gleichermaßen zugänglich sein soll, sondern den (sich wandelnden) Verständnis- und Fragehorizonten der Fachgemeinschaft bzw. Wissenschaft. Individuelle Suchanfragen werden so zu potentiellen Indikatoren für fachspezifische Informationsinteressen. ${ }^{41}$

Somit gerät das information behaviour von Fachgruppen als Kriterium für die Ausgestaltung der Informationsaufbereitung und -verdichtung in den Blick. Als Methode hat sich hier die vor allem von Birger Hjørland vertretene domain analysis etabliert, die davon ausgeht, dass Erkenntnis und Information an eine Wissensdomäne gebunden sind. Sie interessiert sich nicht für den einzelnen User, seine psychologische und kognitive Verfasstheit und individuelles Informationsverhalten, sondern für disziplinäre Usergruppen, die von ihnen kommunikativ und interaktiv konstituierten knowledge domains und deren Informationsstrukturen. Als Forschungsfelder, die hieraus zu generieren sind, benennt Hjørland die Entwicklung fachspezifischer Klassifikations- und Indexierungssysteme, die fachspezifische Evaluation des Anwendungsbereichs und der Qualität von Datenbanken sowie die Ermittlung fachspezifischer Indexierungsprinzipien. Letztlich müsse es das Ziel sein, „to examine the needs for specific information services in concrete domains (such as medicine, music and psychology) or by concrete target groups such as high school students". ${ }^{42}$

Von hier ausgehend, sollten gerade auch Rezensionen als Gegenstand einer empirischen, praxisorientierten Fach-

40 Kleineberg 2012.

41 Allerdings ist auch die umgekehrte Strukturierung $\mathrm{zu}$ sehen, indem die vorherrschende Art und Weise der Wissensrepräsentation und -klassifikation, die auch kulturübergreifenden Entwicklungslogiken folgen, Auswirkungen auf die Weltbildstrukturen der jeweiligen Wissenschaft haben; ebd., S. 131-133.

42 Hjørland 2000, S. 515. informationsforschung stärker in den Blick genommen werden. Zunächst ist zu klären, für welche Wissenschaften und Fachrichtungen Rezensionen welche Rolle spielen. Methodisch ausgehend von der durch die Informationshermeneutik herausgearbeiteten domain analysis könnte mittels Befragungsmethoden oder apparativer Messungen ${ }^{43}$ nach einem wissenschafts- oder fachspezifischen review behaviour als Teil des information seeking behaviour gefragt werden.

Es steht zu vermuten, dass die klassischen Buchwissenschaften fundamental anderen Bedürfnissen nachgehen als die typischen Zeitschriftenwissenschaften. Aktuelle Forschungsergebnisse werden in den Naturwissenschaften viel weniger in Büchern, sondern in Zeitschriften und zumeist online publiziert. Rezensionen spielen daher, so ist zu erwarten, weder als Indikations- und Referenzeinheiten noch als Träger sozialen und kulturellen Kapitals jene wichtige Rolle, die ihnen dort zukommt, wo das Buch nach wie vor nicht nur für wissenschaftliche Qualifikationsarbeiten, sondern auch für die Ergebnisse von Tagungen und Forschungsprojekten das gängige Medium ist. Dies wäre vor allem im geistes- und sozialwissenschaftlichen Bereich, aber auch der Theologie der Fall; in den Rechts- und Wirtschaftswissenschaften, aber auch der Medizin wäre hier möglicherweise stärker nach Subdisziplinen zu unterscheiden.

Um zu differenzierten Ergebnissen zu gelangen, bieten sich Modelle des information seeking behaviour an. Der Prozess der Informationsgewinnung wird hier zwischen dem Anfang des Problembewusstseins und einer ersten unsystematischen Informationssuche auf der einen und der Fixierung und Zusammenführung der relevanten Informationen durch den User auf der anderen Seite in mehrere Phasen unterteilt..4 ${ }^{44}$, an welcher Stelle, zu welchem Zweck und mit welchen Folgen werden - ggf. auf fachspezifische Weise - Rezensionen von Wissenschaftlern herangezogen? Welches sind - textstrukturell - die entscheidenden Informationen? Die referierenden Teile, die auch von indikativen und informativen Abstracts übernommen werden können? Oder eher die Kritik?

Es stellt sich auch die grundsätzliche Frage, welche Komponente, welche Eigenart von Referenztexten inner-

43 Vorbild könnten hier die Forschungen von Paul Huntington, Hamit R. Jamali, David Nicholas und Anthony Watkinson sein, die auf breiter empirischer Basis (3 Millionen User) das Sucherverhalten amerikanischer Universitätswissenschaftler nach lokalen, fachspezifischen und anderen Kriterien im Hinblick auf den Gebrauch digitaler Zeitschriften analysiert haben; Nicholas/Huntington/Jamali 2007; Nicholas/Huntington/Watkinson 2006. 44 Womser-Hacker/Mandl 2013. 
halb der Fachinformation für die Erfüllung der aktivierenden Funktion von DE verantwortlich zeichnet. Wird sie allein durch das Referat erfüllt, indem der „User ... auf einen Blick [sieht], ob der Inhalt des kompletten Dokuments sein Informationsbedürfnis befriedigt"? ${ }^{45}$ Dass hier Zweifel angebracht sind, zeigen empirische Forschungen zum abstract behaviour von Wissenschaftlern, die nämlich selbst dann Bedarf an Abstracts sehen, wenn die DBE digital verfügbar und der Volltextsuche zugänglich ist. Dabei wird aber ganz gegen die dominante Forschungsentwicklung hin zu einem „Abstracting als Extracting“ ${ }^{46}$ eben gerade nicht auf die extraktiven, sondern die derivativen Elemente der Abstracts abgehoben, also jene Ergebnisse der Informationsverdichtung, die in Abstraktion im Sinne von Generalisierung und Sinnverknüpfung bestehen. ${ }^{47}$

\section{Schluss}

Rezensionen erfüllen in bestimmten Wissenschaften wichtige Funktionen innerhalb der Fachinformation. Es kann vermutet werden, dass sie für bestimmte Fachgruppen gerade aufgrund der in ihnen institutionalisierten Kritik und kontextuellen Einordnung in die Forschungslandschaft immer wichtiger für die informative Selektion und Diskriminierung von Wissen werden. Dies gilt nicht zuletzt angesichts der wachsenden Menge und der zunehmenden Erscheinungsgeschwindigkeit von Publikationen sowie der zunehmenden Möglichkeiten des Volltextbrowsings, der Verknüpfung mit Verlagsanzeigen und Inhaltsverzeichnissen. Daher sollten Rezensionen von der Informationspraxis stärker als bislang als - wenn auch gewissermaßen extern produzierte - Dokumentationseinheiten verstanden werden. Vor allem für Bibliotheken als Wissenschafts- und Fachinformationseinrichtungen dürfte der Trend, den etwa die Bayerische Staatsbibliothek setzt, ${ }^{48}$ zukunftsweisend sein: die Verknüpfung von online verfügbaren Rezensionen mit der Titelaufnahme des Werkes. In Zeiten zunehmender Ressourcenverknappung sollte freilich die Entscheidung über die fachspezifische Notwendigkeit von solchen Dokumentationsprojekten nicht dem Ermessen einer Bibliotheksleitung auf der Grundlage von Eindrücken überlassen, sondern wissenschaftlich begründet werden; sie ist Teil ihres Qualitätsmanagements. ${ }^{49}$

45 Stock/Stock 2007, S. 381.

46 Hahn 2004, S. 51.

47 Nicholas/Huntington/Jamali 2007; Nicholas/Huntington/Watkinson 2006; Pinto/Lancaster 1999.

48 Landes 2011, S. 671.

49 Dahinden 2013, S. 126-135.
Voraussetzung dafür ist, die Dokumentationseinheit Rezension stärker von dem pragmatischen Kontext der Fachinformation aus zu betrachten. Hierfür ist es aber auch notwendig, dass sich die Informationswissenschaft von dem Kriterium völliger Objektivität des Abstractings löst und die Subjektivität impliziter und explizit Interpretation in zusammenfassenden Texten nicht als Nach-, sondern Vorteil für die Informationsbeschaffung auffasst. Die hermeneutische Wende in der Informationswissenschaft, in deren Rahmen auf Capurros und Hjørlands Hermeneutik der Fachinformation zurückgegriffen werden könnte, stimmt hier grundsätzlich optimistisch.

\section{Literatur}

Acham, Karl: Art. Abstraktion IV, in: Historisches Wörterbuch der Philosophie, hrsg. von Joachim Ritter, Bd. 1, Basel 1971, Sp. 59-63. Albrecht, Wolfgang: Literaturkritik, Stuttgart 2001.

Bardelle, Frank: Formen der kritischen Auseinandersetzung oder: Wie man Urteile über wissenschaftliche Neuerscheinungen verhängt, in: Zeitschrift für Soziologie 18 (1989) S. 54-64.

Capurro, Rafael: Hermeneutik der Fachinformation, Freiburg/München 1986.

Capurro, Rafael: Hermeneutik revisited, in: Tassilo Pellegrini/Andreas Blumauer (Hg.): Semantic Web. Wege zur vernetzten Wissensgesellschaft, Berlin 2006, S. 527-533.

Dahinden, Urs: Methoden empirischer Sozialforschung für die Informationspraxis, in: Kuhlen 2013, S. 126-135.

Daniel, Ute: Kompendium Kulturgeschichte: Theorien, Praxis, Schlüsselwörter, Frankfurt a. M. 2001.

Dzika, Joanna: Implizites Werten in den Rezensionen - am Beispiel von Metapher und Vergleich, in: Lingua ac Communitas 17 (Oktober 2007) S. 87-96, hier: S. 88, in: <URL: http://www. lingua.amu.edu.pl/Lingua_17/lin-8.pdf> (29.02.2016).

Endres-Niggemeyer, Brigitte: Informationsorientiertes Schreiben oder die Produktion von textuell dargestelltem Wissen. Eine Übersicht über den Stand der Kenntnis in den Fachgebieten Wissenschaftssoziologie, Technisches Schreiben, Schreibforschung und Textverarbeitung, in: LDV-Forum 5 (1988) S. 21-37.

Endres-Niggemeyer, Brigitte: Summarizing Information, Berlin 1998. Gaus, Wilhelm: Dokumentations- und Ordnungslehre. Theorie und Praxis des Information Retrieval, 4. Aufl., Berlin/Heidelberg: Springer, 2003.

Hahn, Udo: Die Verdichtung textuellen Wissens zu Information. Vom Wandel methodischer Paradigmen beim automatischen Abstracting, in: Rainer Hammwöhner; Marc Rittberger; Wolfgang Semar (Hg.): Wissen in Aktion. Der Primat der Pragmatik als Motto der Konstanzer Informationswissenschaft. Festschrift für Rainer Kuhlen, Konstanz 2004, S. 51-68.

Hahn, Udo: Automatisches Abstracting, in: Kuhlen (2013) S. 286-301. Hjørland, Birger: Library and information science. Practice, theory, and philosophical basis, in: Information Processing and Management 36 (2000) S. 501-531.

Kaden, Ben: Elektronisches Publizieren, in: Kuhlen 2013, S. 509-519. Kastner, Sebastian; Mandl, Thomas; Straß, Julia Maria: Identifikation von Kundenrezensionen im WWW als Basis eines Crawlers für 
das Opinion Mining, in: Hans-Christoph Hobohm (Hg.): Informationswissenschaft zwischen virtueller Infrastruktur und materiellen Lebenswelten, Glückstadt 2013, S. 384-389.

Klauser, Rita: Die Fachsprache der Literaturkritik. Dargestellt an den Textsorten Essay und Rezension, Frankfurt a. M. u. a. 1992.

Kleineberg, Michael: Die elementaren Formen der Klassifikation. Ein strukturgenetischer Beitrag zur Informationsgeschichte (Berliner Handreichungen zur Bibliotheks- und Informationswissenschaft; Bd. 325), Berlin 2012.

Klingenböck, Ursula: Schablone - Manier - Effekt. Textlinguistische und -pragmatische Überlegungen zur wissenschaftlichen Rezension im Beispiel der MIÖG (1920-1939), in: Mitteilungen des Instituts für Österreichische Geschichtsforschung 121 (2013) S. 87-108.

Kuhlen, Rainer: Informationsaufbereitung III: Referieren (Abstracts Abstracting - Grundlagen), in: ders. u. a. (Hg.): Grundlagen der praktischen Information und Dokumentation, Band 1: Handbuch zur Einführung in die Informationswissenschaft und -praxis, 4. Aufl., München 2004, S. 189-204.

Kuhlen, Rainer u. a. (Hg.): Grundlagen der praktischen Information und Dokumentation, Band 1: Handbuch zur Einführung in die Informationswissenschaft und -praxis, Berlin/Boston 2013.

Landes, Lilian: Die Schriften der Anderen - Rezensionskultur im Umbruch, in: Geschichte in Wissenschaft und Unterricht 62 (2011) S. 669-671.

Liang, Yong: Zu soziokulturellen und textstrukturellen Besonderheiten wissenschaftlicher Rezensionen, in: Deutsche Sprache 19 (1999) S. 289-311.

Nicholas, David; Huntington, Paul; Watkinson, Anthony: The information seeking behaviour of the users of digital scholarly journals, in: Information Processing and Management 42 (2006) S. 13451365.

Nicholas, David; Huntington, Paul; Jamali, Hamit R.: The use, users, and role of abstracts in the digital scholarly environment, in: Journal of Academic Librarianship 33 (2007) S. 446-453.

Pinto, Maria; Lancaster, F. W.: Abstracts and Abstracting in Knowledge Discovery, in: Library trends 48 (1999) S. 234-248.

Ripfel, Martha: Fachtextsorten der Wissenschaftssprachen II: die wissenschaftliche Rezension, in: Lothar Hoffmann; Hartwig Kalverkämper; Herbert Ernst Wiegand (Hg.): Fachsprachen. Ein internationales Handbuch der Fachsprachenforschung und

Terminologiewissenschaft, Berlin/New York 1998, S. 488-493.

Scheutz, Martin: „Turba ist ein ganz gemeiner Kerl“. Rezensionen als Ehrdiskurs am Beispiel der MIÖG (1918-1938), in: Mitteilungen des Instituts für Österreichische Geschichtsforschung 121 (2013) S. 63-86.

Scheutz, Martin; Sommerlechner, Andrea: Einleitung, in: Mitteilungen des Instituts für Österreichische Geschichtsforschung 121 (2013) (Themenheft Rezensionswesen - Erkundungen in einer Forschungslücke) S. 1-7.

Seeger,Thomas: Entwicklung der Fachinformation und -kommunikation, in: Kuhlen (2013), S. 21-36.

Sparck-Jones, Karen; Willett, Peter (Hg.): Readings in information retrieval, San Francisco 1997.

Stock, Wolfgang; Stock, Mechtild: Wissensrepräsentation. Informationen auswerten und bereitstellen, München 2008.

Womser-Hacker, Christa; Mandl, Thomas: Information Seeking Behaviour (ISB), in: Kuhlen 2013, S. 97-108.

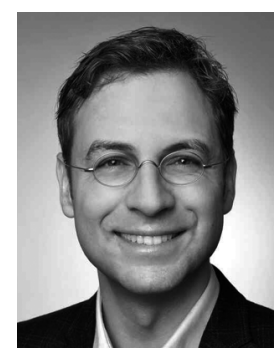

Dr. Jan Brademann

Evangelische Landeskirche Anhalts

Landeskirchliches Archiv

Friedrichstraße 22/24

06844 Dessau-Roßlau

jan.brademann@kircheanhalt.de

Jan Brademann studierte Geschichte, Landesgeschichte und Politikwissenschaft in Halle und wurde 2010 in Münster in mittlerer und neuerer Geschichte promoviert. Seit 2011 ist er Rezensionsredakteur bei der geschichtswissenschaftlichen Informationsplattform H-SozKult. Seit 2015 arbeitet Brademann als Mitarbeiter im Archiv der Evangelischen Landeskirche Anhalts in Dessau und ist eingeschrieben im berufsbegleitenden Masterstudiengang Archivwissenschaft der FH Potsdam. Im Modul „Information und Gesellschaft“ unter Leitung von Prof. Hans-Christoph Hobohm (WS2015/16) entstand der vorliegende Artikel. 